\title{
136. Roller spinning forming regularity
}

\section{Yang Pan', Li Xueguang ${ }^{2}$, Chen Zhe ${ }^{3}$, Yu Yang ${ }^{4}$}

College of Mechanical and Electric Engineering, Changchun University of Science and Technology, Changchun, 130022, China

${ }^{2}$ Corresponding author

E-mail: ${ }^{1386469920 @ q q . c o m,{ }^{2} 174717050 @ q q . c o m,{ }^{3} 13596049542 @ 163 . c o m,{ }^{4} 806942911 @ q q . c o m}$

Received 24 September 2017; accepted 30 October 2017

DOI https://doi.org/10.21595/jme.2017.19187

Check for updates

Abstract. In this paper we use ABAQUS finite element analysis software to design the 3D model. We observed the forming rule of grooved V-shaped pulley during the spinning process, and the impact of rotary working angle and processing temperature on V-belt forming is calculated. The results of this study provide us some theoretical information and pragmatics value for spinning pulley in veritable production.

Keywords: V-pulley, ABAQUS, finite element analysis.

\section{Introduction}

In the automotive industry, pulleys are widely used, in addition to agricultural machinery, washing machines, textile machinery. Spinning pulleys have been successfully promoted and used in large scale for their weight loss, materials saving, high precision, great surface quality, high efficiency and excellent balance performance, replacing the traditional cast iron machining and sheet metal forming [1].

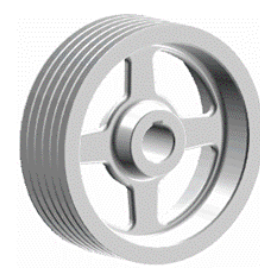

a)

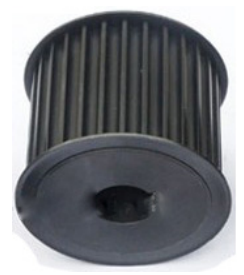

b)

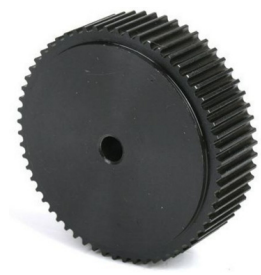

c)

Fig. 1. Several different pulleys

Spinning is the process of the plastic forming of the blank metal by the rotation of the rotary wheel. The new multi-tooth pulley by spin forming, which has a compact structure, high efficiency transmission, has been widely adopted in the transmission. Pre-drawn slabs are preformed and spin-operated to form a multi-tooth new pulley. Spinning requires sufficient axial compression force to prevent loose material and axial flow of materials to ensure radial flow forming. There are many kinds of pulleys that are formed by spinning, but they can be divided into three types according to the forming method, including folding, roll and split. Folding forming method belongs to ordinary spinning. In the process of forming the pulley by the roll forming method, the material is axially displaced, so it belongs to the strong spinning category. The split forming method is divided into two processes when processing the pulley, including the split and forming process [1].

In the plastic forming process of materials, metal spinning is a very important method of processing. In the process of machining, the metal's mobility greatly enhanced, is a very typical large deformation processing methods [2]. Its forming and deformation process is indeed very complex. In its forming process, the blank metal flow displacement is larger, a nonlinear contact between the mandrel and the blank. Finite element simulation cost is relatively low, but also theoretically simulating the spinning process of spinning in actual production, with a certain degree of credibility. This process is presented in a very intuitive form, showing the stress and strain law of the blank metal during the spinning process, and the influence of various process 
parameters on the forming process and the results can be obtained according to the analysis results [4].

With the development of practical experiments and theoretical analysis, the simulation method of deformation process of plastic forming has also made remarkable development, and it has provided considerable help to scientific research and actual production. Finite element simulation is to simulate a process in the actual production, to establish its prototype, through the relevant conditions set as much as possible to simulate the process to facilitate the observation and change the formation of related factors. According to the analysis results obtained under different parameters to analyze the forming rules, so to achieve the understanding of the regularity of the prototype [5].

In this paper, the conventional V-pulley as an example, using roller-squeeze pulley forming method, through the ABAQUS simulation software to establish the spinning belt model and simulate the forming process. The forming results obtained under various parameters are compared to obtain the forming rule of the spinning pulley. It provides a reference for obtaining more accurate pulley-related parameters and is of great significance for the application of spinning pulleys in actual production.

\section{The crafts of spinning process}

\subsection{Spinning principle}

During the spinning process, the blank and the mandrel are fixed together in some way, the mandrel is rotated at the same speed as the blank, and the swivel can feed the blank in either direction. In the process of forming the blanks continue to plastic deformation, and finally spin out the parts to meet the conditions.

\subsection{Classification of spinning process}

According to the process of spinning plastic deformation of the rough situation, spinning technology is generally divided into two kinds of ordinary spinning and strong spinning. Common spinning is called the general rotation, the main categories include: stretch spin, shrinkage spin and expand spin and other forms of processing.

In the spin form, the stretch spin is widely used. Modern machine tool equipment hydraulic profile device, by controlling the wheel feed ratio to adjust the wheel path, pressure, can eliminate the metal radial deformation caused by thinning. In the shrinkage spinning process, the blank is fixed on the spinning machine, so that its radius gradually reduced. The spinning process is to fix the blank to the mandrel with the spindle rotating, and the rotating wheel moves axially along with the rotation of the blank. Expand Spinning is that the hydrodynamic force on the spinning machine causes the preformed blank to radially symmetrically expand. Most of the parts formed by the form of expand spinning are thin-walled components such as drum agitators, wheels and so on.

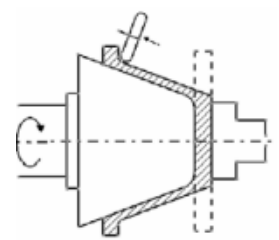

a) Spinning of the Cone pieces

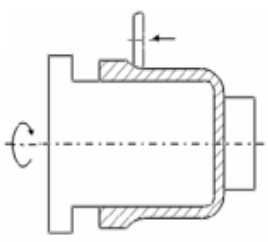

b) Spinning of the cylindrical member

Fig. 2. The classification of strong spinning

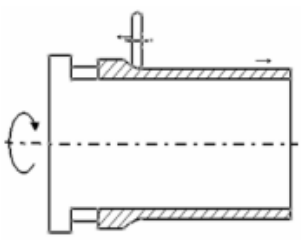

c) Spinning of the tubular part

Strong spinning, also known as thinning spinning. In the forming process, the thickness and shape of the blank have undergone a corresponding change, that is, volume deformation. According to the difference of the shape and the ways of plastic deformation of the workpiece, 
strong spinning is generally divided into tapered parts and cylindrical parts of the strong spinning. According to the direction of rotation of the metal and the direction of the same wheel or not, can be divided into two kinds of positive spinning and anti-spinning. The former refers to the direction of the same, the latter is the opposite direction. The positive spinning is used to spin.

Based on the temperature in the spinning process, the spinning is divided into two types: cold spin and hot spin. The so-called cold spin, that is, the temperature during the spinning process at room temperature, generally used for some material plastic deformation of the workpiece is very good. Different from the cold spin, hot spin refers to the spinning in the heating, in order to achieve the best plastic deformation of the blank in the case of spinning, for the deformation of the larger resistance and plastic deformation of the workpiece material is more applicable.

\section{The roller spinning machine}

Vertical CNC V-belt spinning machine is the manufacture of V-pulley special equipment. Machine design novel, reasonable structure, advanced control, reliable, suitable for the formation of mass production of professional plant. V-pulley-specific equipment with $30 \mathrm{t}$ and $60 \mathrm{t}$ vertical CNC pulley spinning machine. VPS-30C vertical CNC pulley spinning machine by the host, throttle control pump station, volume control pump station, electrical cabinet, mini control cabinet and other components. The main technical parameters are shown in Table 1.

Table 1. Main technical parameters

\begin{tabular}{|c|c|c|c|c|c|}
\hline $\begin{array}{c}\text { Axial } \\
\text { pressure } \\
\left(P_{z}\right)\end{array}$ & $\begin{array}{c}\text { Radial } \\
\text { pressure } \\
\left(P_{r}\right)\end{array}$ & $\begin{array}{c}\text { Main pump } \\
\text { power }\end{array}$ & $\begin{array}{c}\text { Speed pump } \\
\text { power }\end{array}$ & Spindle speed & Processing range \\
\hline $380 \mathrm{kN}$ & $125 \mathrm{kN}$ & $22 \mathrm{~kW}$ & $6.2 \mathrm{~kW}$ & $100-500 \mathrm{r} / \mathrm{min}$ & $\phi_{\max }=280 / \mathrm{t}=8 \mathrm{~mm}$ \\
\hline
\end{tabular}

\section{The spinning process and the analysis of results}

\subsection{Building the model}

The ABAQUS software was used to establish the preformed finite element model. In the simulation process, the blank is assumed to be elasto-plastic deformation, the core mold and the rotating wheel are assumed to be analytical rigid body. The outer surface of the core mold is in contact with the inner surface of the workpiece. The contact and separation between the two are judged by the contact theory. The mandrel plays the role of material diversion in the process of deformation.

The friction between the core and the workpiece is calculated according to the law of Coulomb friction, the friction coefficient is 0.3 . And the friction between the surface of the rotor and the workpiece is negligible in this paper. The mandrel drives the blank at the same time to rotate, and the rotor rotates both the axial and the radial feed to the blank so that the metal flow of the blank material causes the plastic forming of the blank. In the pulley spinning, the blank, the core mold and the rotating wheel are both dynamic contact, and the rough metal material flows continuously, thus selecting ABAQUS / Explicit dynamic explicit module to simulate the process.

Pulley materials often used gray cast iron, steel, aluminum or engineering plastics. Gray cast iron is the most widely used, when $v \leq 30 \mathrm{~m} / \mathrm{s}$, with HT200; when $v \geq 25-45 \mathrm{~m} / \mathrm{s}$, it is appropriate to use ductile iron or cast steel [6]. In this paper, 45 steel is selected as the V pulley material. The properties of the pulley material and the mechanical properties are shown in Table 2.

For the sake of calculation, the following assumptions are made for the model:

1) The workpiece material is homogeneous and isotropic;

2) The effect of gravity and inertia on the forming is neglected.

The rotary wheel should have sufficient rigidity, hardness, strength, abrasion resistance, dimensional accuracy, reasonable structural shape and smooth working surface, so the wheel material is selected as $\mathrm{Cr} 12 \mathrm{MoV}$. According to the pulley spinning process requirements, the 
rotating wheel is selected, the schematic diagram of the wheel shape is shown in Fig. 3. The radius of the wheel is $30 \mathrm{~mm}$, the thickness of $10 \mathrm{~mm}, \gamma_{\rho}$ for the rounded fillet radius, $\alpha$ for the rotary wheel operating angle.

Table 2. Properties of the pulley material and mechanical properties

\begin{tabular}{|c|c|c|c|c|c|}
\hline $\begin{array}{c}\text { Pulley } \\
\text { material }\end{array}$ & $\begin{array}{c}\text { Density } \\
\left(\mathrm{g} / \mathrm{cm}^{3}\right)\end{array}$ & $\begin{array}{c}\text { Elastic modulus } \\
(\mathrm{GPa})\end{array}$ & $\begin{array}{c}\text { Poisson's } \\
\text { ratio }\end{array}$ & $\begin{array}{c}\text { Yield strength } \sigma_{b} \\
(\mathrm{MPa})\end{array}$ & $\begin{array}{c}\text { Tensile strength } \sigma_{s} \\
(\mathrm{MPa})\end{array}$ \\
\hline 45 steel & 7.85 & 210 & 0.31 & 355 & 600 \\
\hline
\end{tabular}

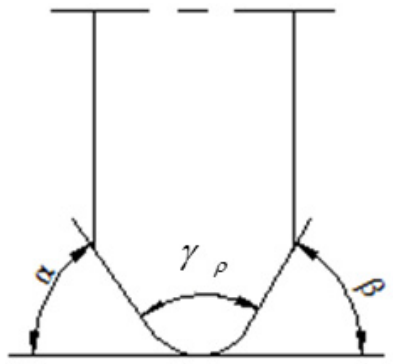

Fig. 3. Schematic diagram of the wheel shape

In the finite element numerical simulation analysis, the grid division has great influence on the analysis speed and the result. In the process of plastic deformation of the blank material, the occurrence of excessive deformation led to the finite element numerical simulation does not converge, thus forming a reasonable analysis results. Therefore, the use of reduced integral hexahedron element division grid, that is, C3D8R partition grid. According to the pulley spinning process, the spinning geometry model is established. As shown in Fig. 4. The three-dimensional finite element model is shown in Fig. 5.

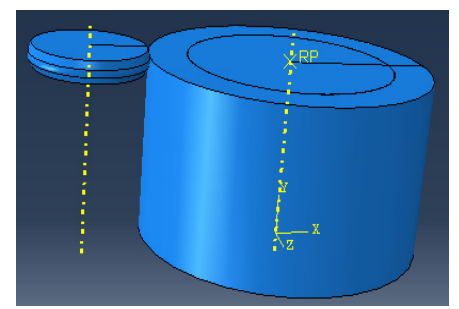

Fig. 4. Spinning geometry model

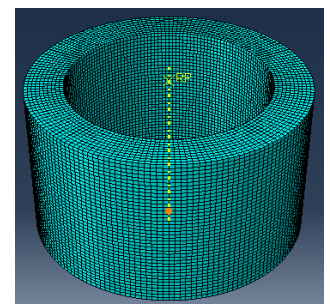

Fig. 5. Three-dimensional finite element model

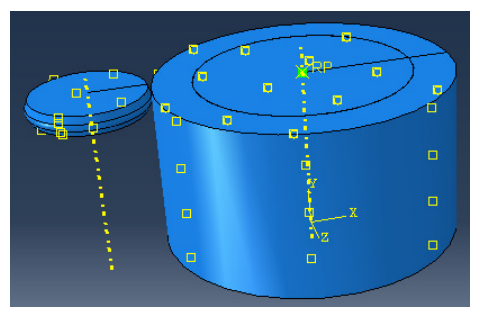

Fig. 6. The geometry of the contact

In the process of pulley spinning, in order to obtain the pulley with the condition, the blank and the mandrel rotate at the same speed, and the rotating wheel must simultaneously move in both axial and radial directions. In the ABAQUS finite element model, the core and the blank are fixed together in the form of tie (the blank and the clamping device are bound together, in the forming process, to eliminate the relative rotation between them, you can eliminate the displacement of the rigid body); And apply a rotational load to the reference point of the mandrel, 
the mandrel drives the blank to rotate, thereby realizing the rotation of the blank around the main shaft. The geometry between the rotary wheel and the workpiece is defined as the general contact relationship.

\subsection{The influence of the working angle of the rotary wheel on the forming of the pulley}

In order to obtain high-quality processed products must be appropriate to select the rotary wheel working angle $\alpha$, fillet radius $\gamma_{\rho}$, wall thickness reduction rate $\psi_{t}$, wheel feed rate $V_{f}$, processing temperature and other parameters. In order to analyze the effect of the working angle $\alpha$ on the spinning process, the following analytical scheme is initially developed. See Table 3.

Three groups of parameters under the analysis shown in Fig. 7.

Table 3. Analysis program

\begin{tabular}{|c|c|c|c|c|}
\hline & Working angle $(\alpha)$ & $\begin{array}{c}\text { Working fillet } \\
\text { radius }(\mathrm{mm})\end{array}$ & $\begin{array}{c}\text { Wall thickness } \\
\text { reduction rate } \psi_{t}\end{array}$ & Rotary federate $V_{f}$ \\
\hline $\begin{array}{c}\text { The first } \\
\text { group }\end{array}$ & $10^{\circ}$ & 10 & $20 \%$ & 0.8 \\
\hline $\begin{array}{c}\text { The second } \\
\text { Group }\end{array}$ & $20^{\circ}$ & 10 & $20 \%$ & 0.8 \\
\hline $\begin{array}{c}\text { The third } \\
\text { group }\end{array}$ & $30^{\circ}$ & 10 & $20 \%$ & 0.8 \\
\hline
\end{tabular}

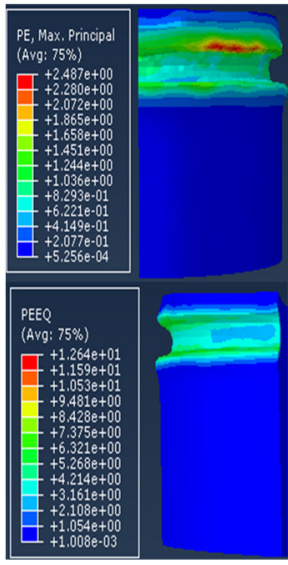

a) Rotary working angle is $10^{\circ}$
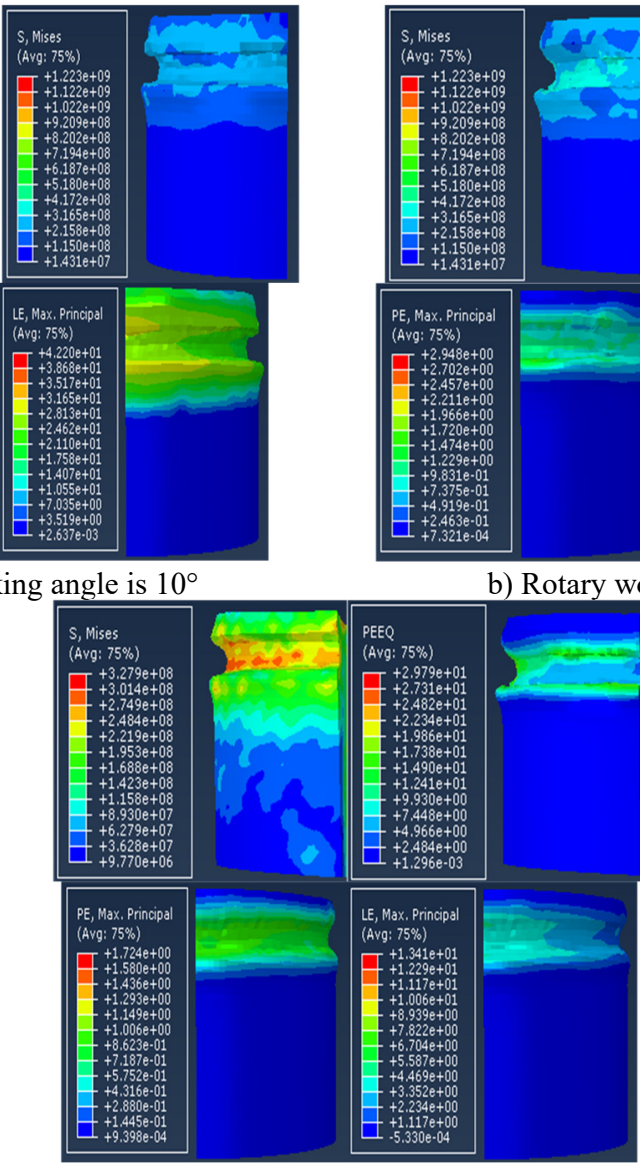

c) Rotary working angle is $30^{\circ}$

Fig. 7. The analysis of results

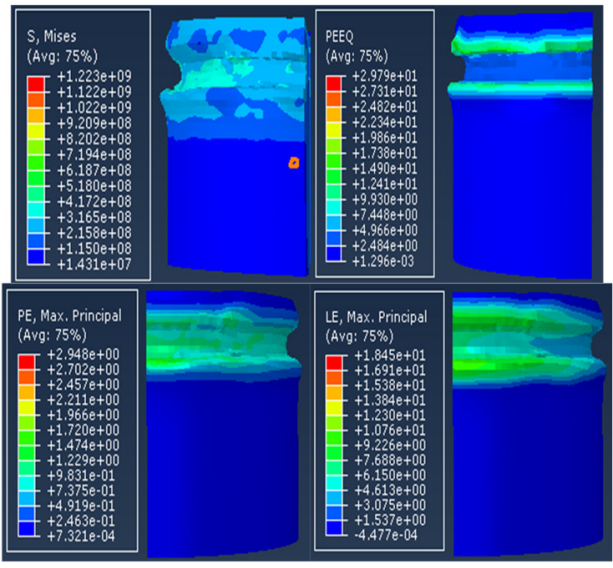

b) Rotary working angle is $20^{\circ}$ 
As can be seen from Fig. 7, as the rotational angle $\alpha$ increases, the higher the uplift of the blank metal in contact with the front end of the rotor, resulting in non-stable flow of the metal. Therefore, the working angle of the rotary wheel during the spinning process should be smaller in a certain range, that can achieve the purpose of reducing the bulging of the rough surface and the irregular flow of the metal.

\section{Conclusions}

For the pulley forming technology, the spinning application is quite extensive. Based on the rigid plastic finite element theory, the ABAQUS finite element analysis software is used to simulate the spinning process. In the spinning process, the parameters that affect the quality of the pulley include the working angle $\alpha$, the fillet radius $\gamma_{\rho}$, the wall thickness reduction rate $\psi_{t}$, the rotary feed rate $V_{f}$, the processing temperature and so on. In this paper, the influence of rotary working angle on spinning is analyzed.

\section{References}

[1] Zhao Yunhao, Li Yanli Spinning Technology and Application. Mechanical Industry Press, Beijing, 2007.

[2] Liu Jiansheng, Chen Huiqin, Guo Xiaoxia Metal Plastic Processing Finite Element Simulation Technology and Application. Metallurgical Industry Press, 2003.

[3] Zhuang Zhuo ABAQUS Nonlinear Finite Element Analysis and Examples. Science Press, Beijing, 2005.

[4] Shen Guozhang Study on Deformation Stability of Belt Wheel Hub in Spinning Forming. Master's Thesis, Hefei University of Technology, 2014.

[5] Zhang Tao Spinning Process. Chemical Industry Press, Beijing, 2009.

[6] Wu Zongze, et al. Mechanical Parts Design Manual. Machinery Industry Press, Beijing, 2013. 